Saudi Journal of Oral and Dental Research

Abbreviated Key Title: Saudi J Oral Dent Res

ISSN 2518-1300 (Print) |ISSN 2518-1297 (Online)

Scholars Middle East Publishers, Dubai, United Arab Emirates

Journal homepage: https://saudijournals.com

\title{
Orthodontists' Perspective on Timing of Functional Appliance Use in Saudi-Based Practices
}

Mohammed K. Badri ${ }^{1 *}$

${ }^{1}$ Department of Pedodontics \& Orthodontics, College of Dentistry, Taibah University, Madinah, Saudi Arabia

DOI: $\underline{10.36348 / \text { sjodr.2021.v06i04.001 }}$

| Received: 26.02.2021 | Accepted: 29.03.2021 | Published: 02.04.2021

*Corresponding author: Mohammed K Badri

\section{Abstract}

Introduction: Literature revealed many studies were concerned with the ideal timing of skeletal discrepancies treatment regardless the type of appliance used. Aim: This study aimed to investigate perspective of orthodontists toward ideal timing for different treatment procedures with functional appliances and explore their association with the demographic background in Saudi-based offices. Materials and methods: In this observational analytical cross-sectional study a webbased questionnaire was distributed among members of the Saudi Orthodontic Society. Questionnaire involved background questions and closed-ended questions regarding timing of treatment, protocol of approaching, and length of treatment with functional appliances. Data analyzed in frequency tables and the use of association tests with significant level of $\mathrm{p}<0.05$. Results: One hundred and four orthodontists (65\% males, 35\% females) completed the distributed questionnaire. Over 50\% of orthodontists preferred to align the dentition after functional treatment. Majority (81.7\%) preferred functional treatment of Class II deformities at the stage of late mixed dentition. Orthodontists were about equally divided in their management in terms of the phases of Class II treatment. Orthodontists revealed a high preference rate for early intervention by functional appliances during both stages of deciduous and early mixed dentition $(81.8 \%)$. Accordingly, their treatment was usually pursued in two phases. The length of treatment with functional appliances in Class II cases was mainly preferred in the range of 7 to 12 months $(61.5 \%)$ while in Class III a dominant preference for the period of 7 to 18 months of treatment (74\%). Conclusion: Orthodontists' training background was strongly associated with many treatment decisions using functional appliances especially in management of Class II cases.

Keywords: Functional appliances, Treatment Timing, Treatment duration.

Copyright () 2021 The Author(s): This is an open-access article distributed under the terms of the Creative Commons Attribution 4.0 International License (CC BY-NC 4.0) which permits unrestricted use, distribution, and reproduction in any medium for non-commercial use provided the original author and source are credited.

\section{INTRODUCTION}

There is an existing controversy about the ideal timing of approaching growing patients with functional appliances and orthodontists have different reasonings in whether to treat the malocclusion in two phases or one single phase. In the last century, clinicians' opinion such as Angel, Nance, Fisher, and others played an important role in advocating early treatment as soon as dental malocclusion was noticed or as late as it was mixed dentition stage [1]. Recent orthodontic clinical studies and randomized controlled trials, mainly focusing on Class II skeletal malocclusion, revealed that there is no big difference in jaw or dental relations between two-phase (early) or one-phase (late) treatment [2-7]. Although early intervention has increased the burden of lengthy treatment period, didn't reduce the need for extraction nor orthognathic surgery during the second treatment phase and resulted in less than optimal occlusion [5, 7], many children still receiving early treatment for esthetic, psychological, or functional reasons and depending on the judgment of the treating orthodontist and preference of the patients and their guardians $[8,9]$.

Clinical studies that investigated the optimal treatment timing for Class III malocclusions showed a popular consensus of early treatment with some variations. Several studies reported better improvement of treatment results when the intervention was as early possible in the deciduous stage of dentition comparing to later dental developmental stages [10-15]. Supporting of early Class III correction was reasoned to prevent worsening of the malocclusion in later dental stages and to minimize or avoid further treatment later on $[13,16$, 
Mohammed K. Badri; Saudi J Oral Dent Res, Apr, 2021; 6(4): 139-147

17]. Some investigations demonstrated no differences in the outcomes between young and old age groups of treated Class III discrepancies as long as the patients encountered their pubertal growth spurt during treatment and the clinicians could maintain the achieved results over time $[8,18,19]$.

Surveys and studies of orthodontists' opinions and practices for the ideal time of intervention for different dental discrepancies showed diverse preferences for different populations. Orthodontists from US or Italian populations revealed their preference toward early treatment for Class II correction during the primary or early mixed dentition followed by a later stage of treatment $[8,20]$. Similarly, orthodontists in Finland and Saudi Arabia approached Class II cases early with the exception for severer cases in which they showed preference to handle them in late mixed or permanent dentition [21, 22]. Other populations such as the Turkish, Australian and Brazilian practitioners displayed their inclination toward postponing Class II intervention to the late mixed or permanent dentition stages $[20,23,24]$. In consistent with clinical trials, majority of the surveys showed that the orthodontic community lean toward the idea of early treatment for Class III cases not later than the stage of early mixed dentition [8,20-24].

The literature revealed many studies were concerned with the ideal timing of skeletal discrepancies treatment regardless the type of appliance used. Therefore, more specifically the aim of the current study was to investigate ideal timing of different treatment procedures in relation to functional appliances and the association between them and the demographic background of orthodontists practicing in Saudi Arabia.

\section{MATERIALS AND METHODS Study Design}

An observational analytical cross-sectional study was designed to investigate the practice and opinion of orthodontists toward functional appliances timing of treatment.

\section{Study Settings and Population}

Our investigation targeted orthodontists whether specialists or consultants from different nationalities who are currently practicing in various facilities in Saudi Arabia and are members of the Saudi Orthodontic Society (SOS). The SOS is the main society in Saudi Arabia pertaining to facilitate communication between orthodontists with more than 400 active members in the society.

\section{Sample Size Calculation}

Sample size was computed through a webbased sample size calculator (Rosasoft). The estimated sample for the study depended on a 5\% margin error, $95 \%$ confidence level, $50 \%$ response distribution, and a total population of 400 which resulted in a recommended size of 197 .

\section{Data Collection}

A web-based self-conducted questionnaire was distributed to the candidates with the help of the SOS through sending emails to all members of the society which was followed in three weeks period with another email as a reminder.

\section{Exclusion Criteria}

Members of SOS practicing outside of Saudi Arabia or residents of orthodontic training programs were excluded from the current study.

\section{Data Collection Instrument}

This study used a self-conducted questionnaire that was adopted after comprehensive literature review of relative articles and validated surveys [8, 22, 24]. The current questionnaire was revised by four orthodontic consultants for final validation. It was composed of two major sections. The first section concerned with the sociodemographic background including gender, professional experience, place of residency program, area of present practice, and kind of working sector. The second section involved questions in the closed-ended form regarding timing of treatment, protocol of approaching, and length of treatment with functional appliances.

\section{Data Analysis}

Demographic data and preferences toward timing of treatment using functional appliances were described in frequency tables. Analysis for association between different categorical variables was conducted using either Chi-square or Fisher exact test depending on the number of expected frequencies; moreover, the strength of association was verified by the value of Cramer's V. A p-value of less than 0.05 was considered to be statistically significant. The analysis was computed by the Statistical Package for the Social Sciences software (IBM ${ }^{\circledR}$ SPSS $^{\circledR}$ Statistics, Version 23).

\section{Ethical Consideration}

Ethical approval was acquired from the Taibah University College of Dentistry, Research Ethics Committee (TUCD-REC) in Madinah, Saudi Arabia with a reference number TUCDREC / 20190918 / MBadri. All participants were informed of the aim of our investigation and their voluntary right for joining the study. Measures were taken to ensure anonymity of the participants' identities and not to violate their privacy.

\section{RESULTS \\ Demographic background}

From all the approached candidates only one hundred and four orthodontists were able to complete the distributed survey with about $26 \%$ response rate. Table 1 shows the demographic data and background of 
the participants. Big number of the orthodontists were males $(65.4 \%)$ with an experience of practice equals to 10 years or less. The location of orthodontics training of the participants revealed the variety and distribution of training programs over different countries and geographic areas. Saudi training programs constituted more than $50 \%$ of the Middle Eastern training. Orthodontists were mainly practicing in the private sector $(67.3 \%)$ with a distribution over the central, western, and eastern areas of Saudi Arabia.

Table-1: Demographic background

\begin{tabular}{ll}
\hline Variable & $\mathbf{n}(\%)$ \\
\hline Gender & \\
Male & $68(65.4)$ \\
Female & $36(34.6)$ \\
\hline Years of experience & \\
10 or less & $61(58.7)$ \\
More than 10 & $43(41.3)$ \\
\hline Orthodontic training & \\
Asia & $22(21.2)$ \\
Europe & $26(25)$ \\
Middle East & $30(28.8)$ \\
North America & $24(23.1)$ \\
Other & $2(1.9)$ \\
\hline Current practice & \\
Central & $43(41.3)$ \\
Eastern & $21(20.2)$ \\
Western & $32(30.8)$ \\
Other & $8(7.7)$ \\
\hline Working sector & \\
Private practice & $70(67.3)$ \\
Teaching Institution & $35(33.7)$ \\
Governmental Hospital & $22(21.2)$ \\
\hline
\end{tabular}

\section{Frequency of functional appliance use}

Two third of the orthodontists reported to use functional appliances routinely in their practice while less than $6 \%$ never used them before. There was more use of removable functional appliances than the fixed type as one fourth of the functional appliances' users reported to never use the fixed type.

\section{Timing of treatment with functional appliance}

In Table- 2 orthodontists revealed their opinion and response toward the time of use of functional appliances based on the type of malocclusion. More than $50 \%$ of participants prefer to align the dentition after finishing treatment with functional appliances. The rest of participants reported to either align the dentition before or during treatment with functional appliance.

Most orthodontists agreed on approaching Class II cases using functional appliances in the stage of late mixed dentition and few preferred to initiate treatment during the early mixed dentition. On the other hand, the preference was the opposite in cases of Class III malocclusion with majority of orthodontists preferred the deciduous and early mixed stages for functional appliance treatment while a decent number of participants preferred the late mixed dentition to intervene.

In regard to Class II cases practitioners were about equally divided in their management in terms of the phases of treatment. 56\% reported to depend on two-phases of treatment with an intervention at early mixed dentition stage with a break period until the final definitive treatment at the permanent dentition is conducted. The other group favored to delay execution of treatment until the period of early permanent dentition considering it as one-phase of treatment. On the other hand, the major bulk of practitioners (92\%) disclosed that they manage cases of Class III as early as possible and eventually followed by the definitive phase at permanent dentition with an overall of two-phase of treatment.

The length of treatment with functional appliances in Class II cases was mainly preferred in the range of 7 to 12 months $(61.5 \%)$ while one fourth of orthodontists revealed their preference for no more than 6 months of treatment and less than $14 \%$ would consider a period of treatment more than 12 months. In regard to Class III cases, there was a random distribution of the results over different time frames: however, with a dominant preference for the period of 7 to 18 months of treatment (74\%). 
Upon considering functional appliances, when do you prefer to align the dentition?

Before using the functional appliance $\quad 22 \quad 21.2$

$\begin{array}{lll}\text { After using the functional appliance } & 59 & 56.7\end{array}$

Simultaneously with the use of the functional appliance

When do you prefer to use functional appliances in Class II cases?

$\begin{array}{ccc}\text { Deciduous Dentition } & 2 & 1.9 \\ \text { Early mixed dentition } & 11 & 10.6 \\ \text { Late mixed dentition } & 85 & 81.7 \\ \text { Permanent dentition } & 6 & 5.8\end{array}$

When do you prefer to use functional appliances in Class III cases?

$\begin{array}{ccc}\text { Deciduous Dentition } & 22 & 21.2 \\ \text { Early mixed dentition } & 63 & 60.6 \\ \text { Late mixed dentition } & 17 & 16.3 \\ \text { Permanent dentition } & 2 & 1.9\end{array}$

What is your protocol of treating Class II malocclusion?

Early intervention followed by a second phase of definitive treatment $\quad 58 \quad 55.8$

$\begin{array}{lll}\text { One phase of intervention at the time of adolescence } & 46 & 44.2\end{array}$

What is your protocol of treating Class III malocclusion?

Early intervention followed by a second phase of definitive treatment $\quad 96 \quad 96.3$

One phase of intervention at the time of adolescence

$8 \quad 7.7$

On average how long do you use /instruct patients to use functional appliances

in Class II cases?

$\begin{array}{lcc}\text { 1-6 months } & 26 & 25 \\ \text { 7-12 months } & 64 & 61.5 \\ \text { 13-18 months } & 10 & 9.6 \\ \text { re than 18 months } & 4 & 3.8\end{array}$

On average how long do you use /instruct patients to use functional appliances in Class III cases?

$\begin{array}{lll}\text { 1-6 months } & 16 & 15.4 \\ \text { 7-12 months } & 47 & 45.2 \\ \text { 13-18 months } & 30 & 28.8 \\ \text { re than 18 months } & 11 & 10.6\end{array}$

\section{Association between timing of treatment and demographics}

Table-3 revealed the significant statistical association between the preferred time of dentition alignment in relation to functional appliances use and the location of orthodontic training $(\mathrm{p}<0.005)$ while no positive associations were found with other orthodontists' demographic background $(\mathrm{p}>0.05)$.

Table-3: Association between timing of dentition alignment relative to functional appliance use and demographics

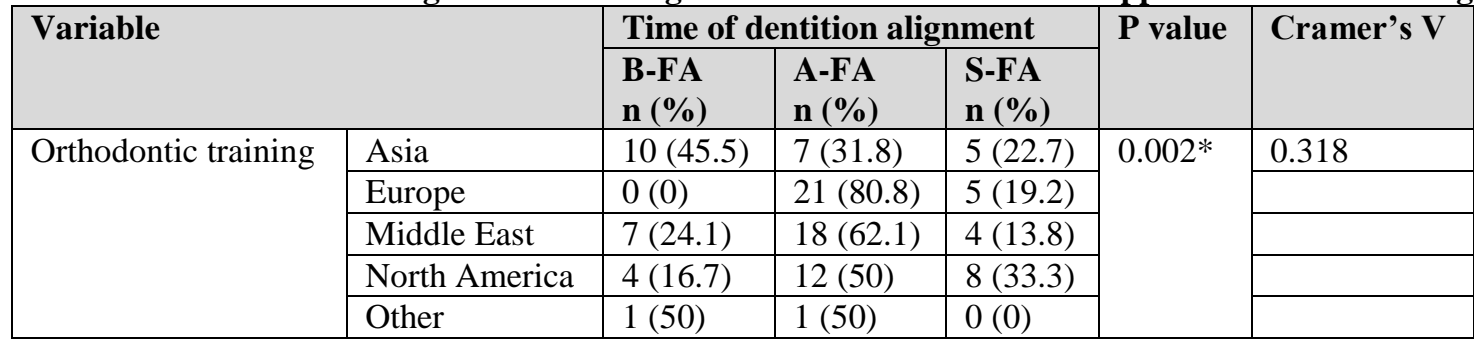

B-FA (Before functional appliance), A-FA (After functional appliance), S-FA (Simultaneous with functional appliance), * Statistically significant, $\mathrm{P}<0.005$

In management of Class II cases, there was a statistically significant association between time of intervention for Class II malocclusion and both location of orthodontic residency $(\mathrm{p}<0.005)$ and area of current orthodontic practice $(\mathrm{p}<0.05)$ (Table-4). The inclination toward treating Class II cases in the late mixed dentition 
was more predominant among orthodontists trained in European programs followed by Middle Eastern programs. Moreover, the preference for intervention in the late mixed dentition was more prevalent among practitioners in Western followed by Eastern areas.
Data revealed no statistically significant association between the preferred time of intervention for Class II and other demographic data including gender, experience, or working sector $(\mathrm{p}>0.05)$.

Table-4: Association between time of intervention for Class II malocclusion and demographics

\begin{tabular}{|c|c|c|c|c|c|c|c|}
\hline \multirow{2}{*}{\multicolumn{2}{|c|}{ Variable }} & \multicolumn{4}{|c|}{ Time of intervention } & \multirow[t]{2}{*}{$P$ value } & \multirow[t]{2}{*}{ Cramer's V } \\
\hline & & & & & & & \\
\hline & & n $(\%)$ & n $(\%)$ & $\begin{array}{l}\text { LiVIU } \\
\text { n }(\%)\end{array}$ & n $(\%)$ & & \\
\hline \multirow[t]{5}{*}{ Orthodontic training } & Asia & $2(9.1)$ & $5(22.7)$ & $15(68.2)$ & $0(0)$ & \multirow[t]{5}{*}{$0.003 * *$} & 0.301 \\
\hline & Europe & $0(0)$ & $0(0)$ & $24(92.3)$ & $2(7.7)$ & & \\
\hline & Middle East & $0(0)$ & $2(6.7)$ & $27(90)$ & $1(3.3)$ & & \\
\hline & North America & $0(0)$ & $3(12.5)$ & $19(79.2)$ & $2(8.3)$ & & \\
\hline & Other & $0(0)$ & $1(50)$ & $0(0)$ & $1(50)$ & & \\
\hline \multirow[t]{4}{*}{ Current practice } & Central & $2(4.7)$ & $6(14)$ & $33(76.7)$ & $2(4.7)$ & \multirow[t]{4}{*}{$0.041^{*}$} & 0.217 \\
\hline & Eastern & $0(0)$ & $2(9.5)$ & $18(85.7)$ & $1(4.8)$ & & \\
\hline & Western & $0(0)$ & $0(0)$ & $30(93.8)$ & $2(6.3)$ & & \\
\hline & Other & $0(0)$ & $3(37.5)$ & $4(50)$ & $1(12.5)$ & & \\
\hline
\end{tabular}

DD (Deciduous Dentition), EMD (Early mixed dentition), LMD (Late mixed dentition), PD (Permanent Dentition).

* Statistically significant, $\mathrm{P}<0.05$, ** Statistically significant, $\mathrm{P}<0.005$

Results of crosstabulation between protocol of Class II treatment and demographic background demonstrated a statistically significant association with location of orthodontic residency $(\mathrm{p}<0.001)$ and type of working sector $(\mathrm{p}<0.05) \quad($ Table-5). Orthodontists trained in Asian programs more likely to lean toward two-phases in treatment of Class II while those trained in North American programs more likely to approach the treatment in one-phase. Furthermore, practitioners working in the private sector more expected to pursue treatment in two-phases; however, those practicing in a teaching institution more expected to conduct treatment in a single phase. Other demographic data such as gender, experience, or area of practice had no significant association with the preferred phases of Class II treatment ( $\mathrm{p}>0.05)$.

Table-5: Association between protocol of Class II treatment and demographics

\begin{tabular}{|c|c|c|c|c|c|}
\hline \multirow{2}{*}{\multicolumn{2}{|c|}{ Variable }} & \multicolumn{2}{|c|}{ Class II protocol } & \multirow[t]{2}{*}{$P$ value } & \multirow[t]{2}{*}{ Cramer's V } \\
\hline & & & & & \\
\hline & & \multirow{2}{*}{$\begin{array}{l}\text { One phase } \\
\text { n (\%) } \\
2(9.1)\end{array}$} & $\begin{array}{l}\text { Two phases } \\
\text { n }(\%)\end{array}$ & & \\
\hline \multirow[t]{5}{*}{ Orthodontic training } & Asia & & $20(90.9)$ & \multirow[t]{5}{*}{$0.000 * *$} & 0.442 \\
\hline & Europe & $14(53.8)$ & $12(46.2)$ & & \\
\hline & Middle East & $14(46.7)$ & $16(53.3)$ & & \\
\hline & North America & $16(66.7)$ & $8(33.3)$ & & \\
\hline & Other & $0(0)$ & $2(100)$ & & \\
\hline \multirow[t]{3}{*}{ Working sector } & Private practice & $28(40)$ & $42(60)$ & \multirow[t]{3}{*}{$0.045^{*}$} & 0.221 \\
\hline & Teaching Institution & $23(65.7)$ & $12(34.3)$ & & \\
\hline & Governmental Hospital & $11(50)$ & $11(50)$ & & \\
\hline
\end{tabular}

* Statistically significant, $\mathrm{P}<0.05$, ** Statistically significant, $\mathrm{P}<0.001$.

Preferences for timing of intervention or protocol of treatment for Class III malocclusion disclosed no statistical association with practitioners' different demographic variables $(p>0.05)$ indicating that selections in reference to demographics were completely based on chance.

\section{DISCUSSION}

Orthodontic practitioners in Saudi-based practices revealed a decent use of functional appliances on a routine basis for treatment of different skeletal discrepancies. Thus, their preferences for the ideal timing, length, and phases of treatment with functional appliances were examined in this cross-sectional study.
Findings from our study displayed the preference of orthodontists toward the order of dentition alignment in relation to functional appliances use. The biggest bulk (57\%) preferred to finish the stage of alignment before starting treatment with functional appliances. Furthermore, a moderately strong association was revealed between such preference and residency training of participants. Orthodontists trained in Asia programs were more likely to start alignment before functional appliance treatment while residents of North American training programs were more likely to pursue alignment concomitantly with functional appliance use than orthodontists of other training programs. Literature shows that treatment of growing patients with functional appliances is most commonly 
followed by a comprehensive fixed appliance treatment in the permanent dentition to align and establish occlusal stability [7, 18, 25-27]. In addition, there are several factors that play a role in determining the order of dentition alignment and functional appliance use including the type of appliance and phases of treatment. Unlike removable functional appliances, many fixed types can be combined with orthodontic brackets and align the dentition simultaneously [28]. Occasionally, early phase of dentition alignment could precede the use of functional appliance in order to align the incisors and facilitate later treatment in a second phase [6, 24]. Moreover, when treatment initiated late in the permanent dentition as one-phase there is a decent chance to not use a functional appliance and depend only on a fixed mechanotherapy treatment [7].

In the current study, treatment of Class II discrepancies was mainly preferred during the stage of late mixed dentition by more than $80 \%$ of practitioners while around $10 \%$ indicated their preference for intervention at the early mixed dentition. Interestingly, the timing of intervention demonstrated a moderate strength of association with the practitioners' orthodontic residency training and location of current practice. Most previous programs were associated with treatment of Class II in the late mixed dentition; however, practitioners trained in Asian programs more likely to initiate treatment during the early mixed dentition than others. In consistence with our findings, Kiyak et al., Miles, and Kaieda et al., demonstrated similar tendency for Class II treatment during the late mixed or the permanent dentition at most for Turkish, Australian, and Brazilian clinicians, respectively [20, $23,24]$. On the other hand, many orthodontists from USA, Italian, Finnish, or Saudi populations contradicted our results and revealed their preference for primary or early mixed dentition stage for correction of Class II discrepancies [8, 20-22].

Orthodontists in this study were split into two groups in their protocol of approaching Class II treatment, about half of them $(56 \%)$ preferred to manage it in two phases while the other group preferred the management in a single one phase during adolescence. Most investigations and clinical trials highlighted that there is no difference in the final outcomes obtained between early (2-phase) or late (1phase) treatment of Class II malocclusion and the benefits gained from early intervention are transient and minimal with no clinical significance [2-7]. Therefore, it has been recommended to delay treatment until the stage of late mixed or early permanent dentition. However, other clinical trials advocated an early intervention, usually during the early mixed dentition, more specifically for severe cases of Class II malocclusion with the aim of reducing the severity, prevent future deterioration, and decrease the length of further treatment or eliminate it; nevertheless, stability of the obtained results was questioned [17, 29]. Keeling et al., revealed in their clinical trial that the skeletal correction achieved with early treatment was stable for a year following the end of treatment while relapse of dental treatment [30], and inferior occlusal stability were reported [7].

Delaying functional treatment in one phase toward the late mixed or permanent dentition usually accompanied with the benefits of immediate comprehensive treatment at the end of skeletal correction, reducing the length, cost and number of appointments of the overall treatment. Those dental stages usually coincide with the pubertal growth spurt in which all of the improvements are taking place. Targeting the pubertal spurt of growth through seeking indicators of skeletal maturity revealed to be the wise approach by most recent studies regardless of the patients' dental age [25-28, 31,32]. Other factors such as practitioners' practice management and the average age of patients seen in the practice could affect the number of phases with functional treatment. Practitioners with high numbers of young patients lean toward early treatment and two-phase approach [2, 8]. Our results revealed the tendency of orthodontists in private practice toward two-phase protocol in management of Class II while those in teaching institutions tend more toward treating in one-phase. In addition, graduates of North American based programs have a practice involving more one-phase of treatment than the others. A reasonable explanation could be the high preference of those practitioners for treatment using fixed functional appliances which usually used during early permanent dentition.

Regarding Class III discrepancies, orthodontists in this study revealed a high preference rate for early intervention by functional appliances during both stages of deciduous and early mixed dentition $(81.8 \%)$; nevertheless, a considerable rate of participants around $16 \%$ leaned toward late mixed dentition intervention. As a result, treatment was favored by majority to be pursued in two phases based on the early intervention. Opinions of orthodontists from different surveys were homogenous with our findings as early stages of deciduous and early mixed dentitions were recommended for intervention for anterior and posterior crossbites correction [8, 20-22]. Gianelly and Grippaudo et al. advocated for early treatment as those cases do not happen to improve overtime and were indicated for immediate intervention $[16,17]$. Yang and Kiyak indicated that the time of treatment was irrelevant if the results were remained stable over the long term [8].

Clinical studies that investigated the timing of functional appliance treatment in Class III patients demonstrated contentious and variable results. Some studies that started treatment during the deciduous or early mixed stage (around 5-8 years) showed an improvement in the skeletal changes on the level of the 
maxilla and mandible [11, 33-35] while other studies did not reveal any effect on the maxilla with only redirection of mandibular vertical position for the correction of discrepancy [36, 37]. Difference in results could be due to the short length of time in using the appliance (around 1 year) comparing to the longer treatment duration ranging $2-3.5$ years in the group with skeletal changes. Our study showed that $74 \%$ of orthodontists preferred longer duration of functional treatment for Class III cases in the range of 7 to 18 months.

Skeletal effects from functional treatment reported to be more effective as early as the intervention was possible. Intervention at deciduous dentition had greater skeletal improvement than mixed dentition; moreover, treatment in mixed dentition had more dentoalveolar effect [11, 15, 38]. Evidence from a recent systematic review concluded that Frankel-III functional appliance more reasonably to restrict mandibular growth and not enough evidence supports its effect on maxilla [39]. However, studies that met the criteria for the review showed a majority of intervention at the early mixed or late mixed dentition and no studies involved intervention at deciduous stage.

Our findings revealed preference of most orthodontists $(86.5 \%)$ for functional treatment of Class II cases to be in a period no longer than 12 months. Length of treatment could vary depending on the type of appliance used but recommendations referred to a period in the range of 1.5 to 2 years $[6,18]$. Some devices such as Twin Block could be used for shorter period of time and usually used for a period around 1 year [4, 40, 41]. Moreover, MARA fixed appliance was recommended to be used for at least 1 year [28]. However, duration of treatment could vary based on the results to be achieved and time of intervention. On the other side, our results for Class III cases revealed a preference of longer period of functional treatment in the range of 7 to 18 months. Clinical studies that used functional devices such as mandibular retractor or Frankel-III demonstrated a minimum of 2.5 years of active treatment for the achievement of stable skeletal effects [33-35] while studies of shorter periods reported no skeletal improvement with more of dentoalveolar effect [36-38].

\section{CONCLUSION}

- Most orthodontists prioritized functional correction of the skeletal deformity over alignment of the dentition.

- The stage of late mixed dentition was the most preferred timing for functional intervention in Class II skeletal deformities.

- Although treatment preference of Class II cases was mainly toward a late dental stage, the management protocol highlighted in number of treatment phases was not conclusive as there was an even preference for both protocols of intervention.

- Orthodontists leaned toward early functional appliance treatment at stages of deciduous and early mixed dentitions in skeletal Class III cases. Accordingly, their treatment was usually pursued in two phases.

- Treatment duration with functional appliances revealed inclination toward longer periods for Class III discrepancies comparing to Class II.

- Orthodontists' training background was strongly associated with many treatment decisions especially in management of Class II cases.

\section{REFERENCES}

1. Wahl, N. (2006). Orthodontics in 3 millennia. Chapter 12: Two controversies: Early treatment and occlusion. American Journal of Orthodontics and Dentofacial Orthopedics, 130(6), 799-804.

2. Livieratos, F. A., \& Johnston, L. E. (1995). A comparison of one-stage and two-stage nonextraction alternatives in matched Class II samples. American Journal of Orthodontics and Dentofacial Orthopedics, 108(2), 118-131.

3. Ghafari, J., Shofer, F. S., Jacobsson-Hunt, U., Markowitz, D. L., \& Laster, L. L. (1998). Headgear versus function regulator in the early treatment of Class II, division 1 malocclusion: a randomized clinical trial. American journal of orthodontics and dentofacial orthopedics, 113(1), 51-61.

4. O'Brien, K., Wright, J., Conboy, F., Sanjie, Y., Mandall, N., Chadwick, S., Connolly, I., Cook, P., Birnie, D., Hammond, M., Harradine, N., Lewis, D., McDade, C., Mitchell, L., Murray, A., O’Neill, J., Read, M., Robinson, S., Roberts-Harry, D., ... Shaw, I. (2003). Effectiveness of early orthodontic treatment with the twin-block appliance: A multicenter, randomized, controlled trial. Part 1: Dental and skeletal effects. American Journal of Orthodontics and Dentofacial Orthopedics, 124(3), 234-243.

5. Tulloch, J. F. C., Proffit, W. R., \& Phillips, C. (2004). Outcomes in a 2-phase randomized clinical trial of early class II treatment. American Journal of Orthodontics and Dentofacial Orthopedics, 125(6), 657-667.

6. Dolce, C., McGorray, S. P., Brazeau, L., King, G. J., \& Wheeler, T. T. (2007). Timing of Class II treatment: Skeletal changes comparing 1-phase and 2-phase treatment. American Journal of Orthodontics and Dentofacial Orthopedics, 132(4), 481-489.

7. O’Brien, K., Wright, J., Conboy, F., Appelbe, P., Davies, L., Connolly, I., Mitchell, L., Littlewood, S., Mandall, N., Lewis, D., Sandler, J., Hammond, M., Chadwick, S., O’Neill, J., McDade, C., Oskouei, M., Thiruvenkatachari, B., Read, M., 
Mohammed K. Badri; Saudi J Oral Dent Res, Apr, 2021; 6(4): 139-147

Robinson, S., ... Worthington, H. (2009). Early treatment for Class II Division 1 malocclusion with the Twin-block appliance: A multi-center, randomized, controlled trial. American Journal of Orthodontics and Dentofacial Orthopedics, 135(5), 573-579.

8. Yang, E. Y., \& Kiyak, H. A. (1998). Orthodontic treatment timing: A survey of orthodontists. American Journal of Orthodontics and Dentofacial Orthopedics, 113(1), 96-103.

9. O’Brien, K., Wright, J., Conboy, F., Chadwick, S., Connolly, I., Cook, P., Birnie, D., Hammond, M., Harradine, N., Lewis, D., McDade, C., Mitchell, L., Murray, A., O’Neill, J., Read, M., Robinson, S., Roberts-Harry, D., Sandler, J., Shaw, I., \& Berk, N. W. (2003). Effectiveness of early orthodontic treatment with the twin-block appliance: A multicenter, randomized, controlled trial. Part 2: psychosocial effects. American Journal of Orthodontics and Dentofacial Orthopedics, 124(5), 488-494.

10. Sakamoto T. (1981). Effective timing for the application of orthopedic force in the skeletal class III malocclusion. American journal of orthodontics, 80(4), 411-416.

11. 11.Baccetti, T. (1998). A retrospective comparison of functional appliance treatment of Class III malocclusions in the deciduous and mixed dentitions. The European Journal of Orthodontics, 20(3), 309-317.

12. Baccetti, Tiziano, McGill, J. S., Franchi, L., McNamara, J. A., \& Tollaro, I. (1998). Skeletal effects of early treatment of Class III malocclusion with maxillary expansion and face-mask therapy. American Journal of Orthodontics and Dentofacial Orthopedics, 113(3), 333-343.

13. Franchi, L., Baccetti, T., \& McNamara, J. A. (2004). Postpubertal assessment of treatment timing for maxillary expansion and protraction therapy followed by fixed appliances. American Journal of Orthodontics and Dentofacial Orthopedics, 126(5), 555-568.

14. Ngan, P. (2005). Early Timely Treatment of Class III Malocclusion. Seminars in Orthodontics, 11(3), 140-145.

15. Toffol, L. D., Pavoni, C., Baccetti, T., Franchi, L., \& Cozza, P. (2008). Orthopedic Treatment Outcomes in Class III Malocclusion. The Angle Orthodontist, 78(3), 561-573.

16. Gianelly, A. A. (1995). One-phase versus twophase treatment. American Journal of Orthodontics and Dentofacial Orthopedics, 108(5), 556-559.

17. Grippaudo, C., Pantanali, F., Paolantonio, E. G., Saulle, R., Latorre, G., \& Deli, R. (2013). Orthodontic treatment timing in growing patients. European Journal of Paediatric Dentistry, 14(3), 231-236.

18. Bishara, S. E., \& Ziaja, R. R. (1989). Functional appliances: A review. American Journal of
Orthodontics and Dentofacial Orthopedics, 95(3), 250-258.

19. Merwin, D., Ngan, P., Hagg, U., Yiu, C., \& Wei, S. H. Y. (1997). Timing for effective application of anteriorly directed orthopedic force to the maxilla. American Journal of Orthodontics and Dentofacial Orthopedics, 112(3), 292-299.

20. Kiyak, H. A., Haluk, I., \& Miotti, F. A. (2004). Orthodontists' perspectives regarding treatment timing: a cross-national study. World journal of orthodontics, 5(1), 40-47.

21. Pietilä, I., Pietilä, T., Pirttiniemi, P., Varrela, J., \& Alanen, P. (2008). Orthodontists' views on indications for and timing of orthodontic treatment in Finnish public oral health care. European Journal of Orthodontics, 30(1), 46-51.

22. Al-Shayea, E. (2014). A survey of orthodontists' perspectives on the timing of treatment: A pilot study. Journal of Orthodontic Science, 3(4), 118.

23. Miles, P. (2013). 2013 survey of Australian orthodontists' procedures. Australian Orthodontic Journal, 29(2), 170-175.

24. Kaieda, A. K., Lima, I. F. P., Scanavini, M. A., Coqueiro, R. S., Pithon, M. M., Rode, S. M., \& Paranhos, L. R. (2017). Perception, knowledge and attitudes of Brazilian orthodontists on the treatment of Class II malocclusions. Anais Da Academia Brasileira de Ciências, 89(4), 2875 2885.

25. Singh, S., Singh, M., Saini, A., Misra, V., Sharma, V. P., \& Singh, G. K. (2010). Timing of myofunctional appliance therapy. The Journal of clinical pediatric dentistry, 35(2), 233-240.

26. Franchi, L., Pavoni, C., Faltin, K., Jr, McNamara, J. A., Jr, \& Cozza, P. (2013). Long-term skeletal and dental effects and treatment timing for functional appliances in Class II malocclusion. The Angle orthodontist, 83(2), 334340.

27. Pavoni, C., Lombardo, E. C., Lione, R., Faltin, K., Jr, McNamara, J. A., Jr, Cozza, P., \& Franchi, L. (2018). Treatment timing for functional jaw orthopaedics followed by fixed appliances: a controlled long-term study. European journal of orthodontics, 40(4), 430-436.

28. Ghislanzoni, L. T., Baccetti, T., Toll, D., Defraia, E., McNamara, J. A., Jr, \& Franchi, L. (2013). Treatment timing of MARA and fixed appliance therapy of Class II malocclusion. European journal of orthodontics, 35(3), 394-400.

29. Tulloch, J. F., Phillips, C., Koch, G., \& Proffit, W. R. (1997). The effect of early intervention on skeletal pattern in Class II malocclusion: a randomized clinical trial. American journal of orthodontics and dentofacial orthopedics, 111(4), 391-400.

30. Keeling, S. D., Wheeler, T. T., King, G. J., Garvan, C. W., Cohen, D. A., Cabassa, S., McGorray, S. P., \& Taylor, M. G. (1998). Anteroposterior skeletal and dental changes after 
early Class II treatment with bionators and headgear. American journal of orthodontics and dentofacial orthopedics, 113(1), 40-50.

31. Faltin, K. J., Faltin, R. M., Baccetti, T., Franchi, L., Ghiozzi, B., \& McNamara, J. A., Jr (2003). Long-term effectiveness and treatment timing for Bionator therapy. The Angle orthodontist, 73(3), 221-230.

32. Baccetti, T., Franchi, L., \& Kim, L. H. (2009). Effect of timing on the outcomes of 1-phase nonextraction therapy of Class II malocclusion. American journal of orthodontics and dentofacial orthopedics, 136(4), 501-509.

33. Tollaro, I., Baccetti, T., \& Franchi, L. (1996). Craniofacial changes induced by early functional treatment of Class III malocclusion. American journal of orthodontics and dentofacial orthopedics, 109(3), 310-318.

34. Miethke, R. R., Lindenau, S., \& Dietrich, K. (2003). The effect of Fränkel's function regulator type III on the apical base. European journal of orthodontics, 25(3), 311-318.

35. Levin, A. S., McNamara, J. A., Jr, Franchi, L., Baccetti, T., \& Fränkel, C. (2008). Short-term and long-term treatment outcomes with the FR-3 appliance of Fränkel. American journal of orthodontics and dentofacial orthopedics, 134(4), 513-524.
36. Biren, S., \& Erverdi, N. (1993). Cephalometric evaluation of maxillary retrognathism cases treated with FR-3 appliance. Journal of Marmara University Dental Faculty, 1(4), 354-360.

37. Baik, H. S., Jee, S. H., Lee, K. J., \& Oh, T. K. (2004). Treatment effects of Fränkel functional regulator III in children with class III malocclusions. American journal of orthodontics and dentofacial orthopedics, 125(3), 294-301.

38. Robertson N. R. (1983). An examination of treatment changes in children treated with the function regulator of Fränkel. American journal of orthodontics, 83(4), 299-310.

39. Yang, X., Li, C., Bai, D., Su, N., Chen, T., Xu, Y., \& Han, X. (2014). Treatment effectiveness of Fränkel function regulator on the Class III malocclusion: a systematic review and metaanalysis. American journal of orthodontics and dentofacial orthopedics, 146(2), 143-154.

40. Baccetti, T., Franchi, L., Toth, L. R., \& McNamara, J. A., Jr (2000). Treatment timing for Twin-block therapy. American journal of orthodontics and dentofacial orthopedics, 118(2), 159-170.

41. Clark W. J. (1988). The twin block technique. A functional orthopedic appliance system. American journal of orthodontics and dentofacial orthopedics, 93(1), 1-18. 